Tomato endophyte bacearch Square
term Organic agricultu
Zeyu Zhang ( $642569725 @ q q . c o m$ )
China Agricultural University
Yabin Zhan
China Agricultural University
Zengqiang Zhang
NWAFU
Youzhou Liu
Jiangsu Academy of Agricultural Sciences
Ting Xu
China Agricultural University
Yuquan Wei
China Agricultural University
Ji Li
China Agricultural University

\title{
Tomato endophyte bacteria diversity under long- term organic agricultural manipulation systems
}

Zeyu Zhang (D 642569725@qq.com )

China Agricultural University

Zengqiang Zhang

NWAFU

Youzhou Liu

Jiangsu Academy of Agricultural Sciences

Ting Xu

Yuquan Wei

China Agricultural University

China Agricultural University

\section{Research Article}

Keywords: Endophytic Bacteria, Organic Farming Systems, Microbial Diversity, Niche

Posted Date: March 12th, 2021

DOl: https://doi.org/10.21203/rs.3.rs-265135/v1

License: (c) (i) This work is licensed under a Creative Commons Attribution 4.0 International License.

Read Full License 


\section{Abstract}

Purpose: Differences were observed in both microbial structure and physicochemical properties were observed in the organic and conventional agricultural systems. However, very little is known about the diversity of endophytic bacterial communities in plants grown in separate manipulation systems. The goal of this work was to get a broader overview of the diversity and dynamics of the tomato endophytic bacteria in the different agricultural manipulation systems.

Methods: The structure of endophyte bacterial communities under different growth periods (seeding stage, flowering stage, fruiting stage and harvesting stage), agricultural manipulation practices (organic and conventional systems) and organ-type (root or stem) were explored by using 16Sr RNA gene profiling in this study.

Results: A total of 2,014,992 part 16 SRRNA gene sequences were obtained. These sequences revealed large-scale functional taxonomy units (OTUs). That is, there are 648 different OTUS in libraries, and 96 OTUs are common. Tomato endophytes consisted mainly of four phylloxera, of which Proteobacteria was the most represented, followed by Formigiotes, bacteriophages, Actinobacteria, Gamma proteobacteria, the most abundant class of proteobacteria, bacteriobacteria, and so on. Proteobacteria are low. General enterobacteriaceae, Vecella, Bacillus, Mesorizobium and Chrysobacterium were shared by all growth periods. Rich endophytic bacterial diversity was observed at the seedling stage (DI), and endophytic bacterial diversity at the flowering stage and fruiting stage was low. Significant difference in endophytic bacterial communities emerged from roots and different host biographical stages, and tomato exerts greater influence on endophyte bacteria compared to organ type (main) agricultural manipulation methods.

Conclusions: Tomato endophyte microbiota have a distinct structure in different growth periods. Bacillus were enriched seeding stage and decreased in the fruiting stage while Mesorhizobium increased during in the fruiting stage. Tomato have distinct endosphere microbiota by comparing beta diversity of microbiota in different growth periods, compared with the manipulation resume and organ type. And a strong correlation was observed between the structure of the microbiota in the whole dataset and soil chemistry which indicated that the soil type and treatment affected the endosphere microbiota of tomato. Organtype (niche) exert more influence on the tomato microbiota compared with manipulation treatments between organic-farming and conventional farming.

\section{Introduction}

Tomato (Solanum glycoprotein) are an important economic crop grown worldwide that are of commercial value and ecological importance. Evidence is increasing that soil microorganisms or those associated with crops play an important role in plant health (Zhao et al. 2016). Plant microorganisms, often representing plant identities and plant second genes, are essential for plant health (Rui et al. 2019). Stabilization of microorganisms to increase plant nutrition and increase resistance to biological and 
aziotic stresses presents one of the few reservoirs that has not been used as an opportunity to address sustainability issues in agriculture (Cao et al. 2004). Therefore, it is important to have a good understanding of the endophytic bacterial communities in tomatoes.

Endophytic bacteria are a diverse group of bacteria that live inside plant tissues; These endophytes can live within cells, in the interstitium, or in the vascular system (Manzotti et al. 2020). Beneficial to the endophytic bacterial host plant, e.g., biological control of growth diseases or plant diseases (Kahremani et al. 2019), in addition, endophytic microorganisms can provide protection against fungal diseases (Vector $J$ et al. 2011). Explaining the structure of endophytic bacteria can help clarify their function and potentially improve performance. Most plant species, including monocotyledonous dicotyledonous plants and endophytic microbiota, have been reported (Constantine et al. 2019). Good agricultural practices, e.g., agricultural microbial vaccines, for example, generally improve the yield of plants produced during organic farming and the growth of suitable plants (Hon et al. 2017). Endophytes microbiota can be affected by a variety of factors such as physical and chemical properties and good farming practices such as organic farming and microbial vaccination. E.g., and plant yields generally excel in organic farming suitable for plant growth (Dong et al. 2019).

A long-term greenhouse test was carried out in 2002 at the Kujo Experimental Station in Hebei Province, China. This experiment includes organic and conventional farming methods for evaluating the endophyte constitution of tomatoes. All three farming methods were under the same schemes like crop rotation, irrigation and plowing, but they differed in fertilization and plant protection management. Differences were found in both the microbial structure and the physicochemical properties (Han et al. 2017). However, little is known about the diversity of endophytic bacterial communities in plants grown under separate manipulation systems, and therefore, the structure (systems and organics) of endosperm bacterial communities under different maneuvers (sowing stage, flowering stage, fruiting stage and harvest stage), and agricultural manipulation practices. Organ type (root or stem) was explored in this study using 16 SRNA gene amplicion sequencing, and the goal of this work was to obtain a comprehensive overview of the diversity and kinetics of tomato endophytic bacteria. Different agricultural manipulation systems.

\section{Materials And Methods}

\section{Growth Conditions and Sample Collection}

Two sampling greenhouse was located in Quzhou (N 34"17"هE 116 $55^{\square} ", 476 \mathrm{~m}$ above sea level). Hebei province, China, of which manipulated by organic and conventional systems since 2002. Plants at four developmental stages, including seedling the stage, flowering stage, fruiting stage, and harvesting stage were chosen during the growth period from March 2019 to August 2019. Five plants were randomly selected for each site at each of the various distinct growth stages, such that 80 plants were sampled in total. Roots were syndicated in Diagenode Bioruptor at low frequency for physical removal of microscopic soil aggregates and attached microbes (Vega et al. 2015). All eighty roots and stem gathered. Surface sterilization was made in previously described (Gu et al. 2020). No bacterial growth 
was detected after plating the roots on $\mathrm{R}_{2} \mathrm{~A}$ agar at $30^{\circ} \mathrm{C}$ for 7 days (Mcpherson et al. 2018). Moreover, no amplification of $16 \mathrm{~S}$ rRNA genes was observed when the water used for the final wash was used as a source of DNA.

\section{Bar-Coded Pyrosequencing}

PCR optimization and IIImina-Hiseq2500 pyrotechnical were performed by the Amplicon Seq Service of the Biomak Biotechnology (China). Briefly, DNA aliquots (10 ng) were PCR-amplified at 94 for $30 \mathrm{~s}$ and then 30 cycles of 94 for $20 \mathrm{~s}, 45$ for $20 \mathrm{~s}$, and 65 for $60 \mathrm{~s}$; with a final extension at 72 for 5 min. V7-V9 hypervariable regions of the bacterial 16S rRNA gene were amplified with the primers $799 \mathrm{~F}$ and $1193 \mathrm{R}$ containing the sequencing adapters and sample-specific barcode (illmina-Hiseq2500 Sciences). The applicants were purified and quantified fluorometrically with the Min Elute kit (Qiagen, Germany).

\section{Sequence Analysis}

The raw 16S rRNA gene sequencing reads were demultiplexed, quality-filtered by fastq version 0.20 .0 and merged by FLASH version 1.2.7 with the following criteria: (i) the 300 bp reads were truncated at any site receiving an average quality score of $<20$ over a 50 bp sliding window, and the truncated reads shorter than 50 bp were discarded, reads containing ambiguous characters were also discarded; (ii) only overlapping sequences longer than $10 \mathrm{bp}$ were assembled according to their overlapped sequence. The maximum mismatch ratio of overlap region is 0.2 . Reads that could not be assembled were discarded; (iii) Samples were distinguished according to the barcode and primers, and the sequence direction was adjusted, exact barcode matching, nucleotide mis match in primer matching (Chen et al. 2020).

\section{Results}

\section{Bacterial Profiling Analysis}

A total of 2,014,992 high-quality, non-plastid, and partial sequences were requested. The number of retained sequences per sample varied from 30,702 to 267,257 (Table. S1), and the rarefaction curves shown in Fig.1, which suggested that these libraries detected a large majority of the endophytic bacterial diversity in the samples used in our study. 648 OTUs were consulted on 80 samples for the bacterial library, with 96 OTUs in common after strict filtering process (Fig.2).

\section{Taxonomic Composition Analysis}

The taxonomic summary of the diversity revealed that the tomato-associated endophyte bacteria mainly comprised four phyla (Fig. 3A), among which Proteobacteria was the most represented (40.63\%), followed by Firmicutes (21.76\%), Actinobacteria (20.3\%) and Bacteroidetes (8.7\%). A total of 253 different genera were identified across all samples, in addition, the relative abundance of bacterial 
classes of the top 10 most abundant genera in different growth periods was shown in Fig. 3B, that is, genera Weissella, Bacillus, Mesorhizobium and Chryseobacterium were shared by all growth periods.

\section{Dynamics of endophytic bacteria in tomato during growth}

The bacterial Shannon diversity index have no significant difference in different growth periods (Fig. 4A P-value cut off $=0.05$ ), while principal coordinate analysis ( $P C O A)$ of Bray-Curtis distance (Fig. 5B, permutations analysis of variance $\mathrm{R}^{2}=0.19, \mathrm{P}<0.001$ ), and endosphere microbiota in four growth periods formed separated clusters in the first two coordinate axes, and all of the profiles indicated that endosphere microbiota changed with the growth of tomato.

PCoA of bacterial microbiota using Bray-Curtis distance for organ sampling and long-term manipulation treatment factor in different growth periods (Fig. 5D-L). Tomato endosphere microbiota in different agricultural manipulation, and organ-type were also distinct. The seeding stage (TI), flowering stage (T2), fruiting stage (T3) and harvesting stage (T4). Seeding stage ( $\mathrm{TI}$ ) datasets selected from the whole dataset were used to analyse the organ-type factor, and root (R) and stem (S) datasets from each growth period were used to analyse the agricultural manipulation treatment factor (FigS.5D-F). The beta diversity and PCoA analyse using Bray-Curtis distance matrices showed that bacterial microbiota of different organ types in four growth periods datasets were separated in the first two coordinate axes (permutations analysis of variance $\left.R^{2}=0.354, P=0.001\right)$, however, treatment factors had no cluster in different root $(R)$ or stem (S) datasets and were separated in the first two coordinate axes (FigS.5D-F), and other root (R) or stem (S) datasets have the similar results (FigS.5G-N): a large variation was found in the endosphere microbiota of tomato with different organ types but a few agricultural manipulation treatments.

\section{Discussion}

During the long history of organic farming experiment from Quzhou county in 2002, three agricultural manipulations were carried out. Here, we explore the endophyte constitution under two farming methods. We reported that the reproductive period is associated with the enrichment of endophytes. In general, the difference from rhizosphere to rhizosphere to phylloxera to endosphere is minimal (Lee et al. 2018), so low concentration and diversity in root and stem endophyte bacterial specimens are understood in this study, and no significance was found in diversity over different time periods (Fig. 2A). The bacteria in the stems come from the environmental soil, air, air or water, which are enriched by microorganisms (Doju et al. 2018). This may explain the greater richness in the roots compared to the stems (Zhuang et al. 2020). Over time, endophytic bacteria from the roots can migrate or be transported to the upper parts of plants (Lamo et al. 2018). In this study, the relatively closed environment provided by the greenhouse may have been another reason for the low diversity of stem bacteria. Furthermore, the bacterial richness and diversity of endophytes vary in different tissues, resulting in greater bacterial diversity in the roots. Similar 
results were reported for other plant hosts such as Arabidopsis, rice and agave species (Paul Schulz et al. 2018).

Additional studies showed that organ-type varieties recruit distinct endophytic microbiota (FigS. 3D-L), suggesting that the niche has a considerable impact on endophyte microbiota establishment, while agricultural manipulation exert limited influence on the constitution endophytes bacteria. As the plant organ-type (niche) explained $29.0 \%$ of the differential abundance in endophytic microbiota, and it will be essential to provide a clue for screening the beneficial microbiota involved in promoting growth and resisting the diseases (Valenzuela et al. 2018).

We may assume that the dynamics of endophytic bacteria communities are due to differing microenvironments in vivo and in vitro under different plant developmental stages, and the similar results showed that structure of endosphere microbiome was affected by the assembly process and plants growing periods (Ye et al. 2018). Most endophytic bacteria are recruited from the soil and rhizosphere bacteria in plant growth processes, maintaining the help with the suppression of pathogen, nitrogen fixation, and this finding was consistent with the previous reports in other plant species, including Arabidopsis, soybean, rice and some of its relatives (Constantin et al. 2019).

Coordination between the host plant and the microbiota is critical to plant growth in natural environments. We found that roots of tomato recruited a higher proportion of Bacillus and Mesorhizoium (Fig. 2), indicating that the Firmicutes probably involved in the nurtrients uptake and plant metabolism, which could explain the majority of beneficial biocontrol-microbiota belong to this phylum in the root environment (Silvina et al. 2018).

Endophytic bacterial diversity studies indicate that taxonomic groups such as Bacteroidetes, Actinobacteria, Acidobacteria, Proteobacteria and Firmicutes are possibly involved in growth by enhancing nutrient acquisition and tolerance to biotic and abiotic stresses (Silvina et al. 2020). In the vegetative tissues (roots, stems and leaves), the bacterial genera Acinetobacter, Enterobacter, Pseudomonas and Pantoea were abundantly present in these samples, albeit in different amounts (Bulgarelli et al. 2012).

In the present study, bacterial communities varied among planting developmental stages. Numerous reports showed that rice genotype in shaping the root microbiota using a limited set of two to four rice varieties (Wei et al. 2020). Salt-accumulating halophyte S. European would depend on microbiota, which allow optimal growth in saline conditions (Sun et al. 2008). Plants not only actively influence the microbiome structure in diverse niches by metabolites (Zhao et al. 2015), but also increase the abundance of beneficial communities in the endosphere at specific growth periods (Romero et al. 2014).

In summary, the present study provides a holistic view of the composition, diversity and influential factors shaping the endophytic bacterial communities associated with greenhouse grown tomato plants. This is still a case study and the knowledge gained in other long-term experiments should be verified to gain a comprehensive understanding of the role of soil microorganisms in plant health, as it is mainly designed 
with each specific environmental significance (Zhao et al. 2016). Some beneficial bacterial strains have been isolated in our laboratories and their proper functions in tomato growth and health will be studied in the future. These efforts will provide an important data base for the further utilization of beneficial bacteria in tomato production.

\section{Conclusion}

Tomato endophyte microbiota have a distinct structure in different growth periods. Bacillus were enriched seeding stage and decreased in the fruiting stage while Mesorhizobium increased during in the fruiting stage. Tomato have distinct endosphere microbiota by comparing beta diversity of microbiota in different growth periods, compared with the manipulation resume and organ type. And a strong correlation was observed between the structure of the microbiota in the whole dataset and soil chemistry which indicated that the soil type and treatment affected the endosphere microbiota of tomato. Organ-type (niche)exert more influence on the tomato microbiota compared with manipulation treatments between organicfarming and conventional farming.

\section{Declarations}

\section{Acknowledgements}

Not applicable.

\section{Authors' contributions}

ZZY designed the research, carried out the experiment, and drafted the manuscript. ZYB improved the writing of the manuscript. ZZQ, LYZ and WYQ revised the manuscript. All authors have read and approved the final manuscript.

\section{Availability of data and materials}

All data analyzed during this study are included in this published article and its supplementary information files.

\section{Funding}

This work was financially supported by the National Natural Science Foundation of China (201503010311304).

\section{Ethics approval and consent to participate}

Not applicable.

\section{Consent for publication}


Not applicable.

\section{Competing interests}

The authors declare that there is no competing interest.

\section{References}

Bulgarelli D, Schlaeppi K, Spaepen S (2012). Structure and functions of the bacterial microbiota of plants. Annual Review of Plant Biology. 64(1):807-838.

Cao L X, Qiu Z Q, You J L (2004). Isolation and characterization of endophytic Streptomyces strains from surface-sterilized tomato (Lycopersicon esculentum) roots.Letters in Applied Microbiology.39(5):425-430.

Constantin M E, Lamo F J D, Vlieger B V (2019). Endophyte-Mediated Resistance in Tomato to Fusarium oxysporum is Independent of ET, JA, and SA. PLOS one.10.

Chen T, Nomura K, Wang X (2020). A plant genetic network for preventing dysbiosis in the phyllosphere. Nature.580(7805).

Dong CJ, Ling L (2019). Bacterial communities in the rhizosphere, phyllosphere and endosphere of tomato plants. PLOS one.14(11): e0223847.

Ghahremani Z, Escudero N, Saus E (2019). Pochonia chlamydosporia Induces Plant-Dependent Systemic Resistance to Meloidogyne incognita. Frontiers in Plant Science.10.

Gu S, Wei Z, Shao Z (2020). Competition for iron drives phytopathogen control by natural rhizosphere microbiomes. Nature Microbiology. 5(8):1-9.

Genitsaris S, Stefanidou N, Leontidou K (2020). Bacterial Communities in the Rhizosphere and Phyllosphere of Halophytes and Drought-Tolerant Plants in Mediterranean Ecosystems. Microorganisms. $8(11)$.

Han, $\mathrm{H}$, Teng, Y, Yang, $\mathrm{H}, \mathrm{Li}, \mathrm{J}$ (2017). Effects of long-term use of compost on $\mathrm{N}_{2} \mathrm{O}$ and $\mathrm{CO}_{2}$ fluxes in greenhouse vegetable systems. Compost Sci. Uti.25(Suppl. 1), S61-S69.

Han Q, Ma Q, Chen Y (2020). Variation in rhizosphere microbial communities and its association with the symbiotic efficiency of rhizobia in soybean. The ISME Journal. 14(8).

Klein E, Ofek M, Katan J (2013). Soil Suppressiveness to Fusarium Disease: Shifts in Root Microbiome Associated with Reduction of Pathogen Root Colonization. Phytopathology. 103(1):23.

Lee S M, Kong H G, Song G C (2019). Disruption of Firmicutes and Actinobacteria abundance in tomato rhizosphere causes the incidence of bacterial wilt disease. The ISME Journal. 
Lamo F J D, Constantin M E, Fresno D H (2018). Xylem Sap Proteomics Reveals Distinct Differences Between R Gene- and Endophyte-Mediated Resistance Against Fusarium Wilt Disease in Tomato. PLOS one. 9.

Li H, Cai X, Gong J (2019). Long-Term Organic Farming Manipulated Rhizospheric Microbiome and Bacillus Antagonism Against Pepper Blight (Phytophthora capsici). Frontiers in microbiology.10.

Manzotti A, Bergna A, Burow M (2020). Insights into the community structure and lifestyle of the fungal root endophytes of tomato by combining amplicon sequencing and isolation approaches with phytohormone profiling. FEMS Microbiology Ecology.2020.

Mcpherson M R, Peng W, Marsh E L (2018). Isolation and Analysis of Microbial Communities in Soil, Rhizosphere, and Roots in Perennial Grass Experiments. Journal of Visualized Experiments. (137).

Rui H, Yang DP (2019). Bacterial Profiling and Dynamic Succession Analysis of Phlebopus portentosus Casing Soil Using MiSeq Sequencing. Frontiers in microbiology.10:1927-1927.

Silvina M. Y. López, Pastorino G N, Antonio J. Fernández-González (2020). The endosphere bacteriome of diseased and healthy tomato plants. Archives of Microbiology.202(1).

Ryan RP, Germaine K, Franks A, Ryan DJ, Dowling DN (2008) Bacterial endophytes: recent developments and applications. FEMS Microbiol Lett 278:1-9.

Romero FM, Marina M, Pieckenstain FL (2014) The communities of tomato (Solanum lycopersicum L.) leaf endophytic bacteria, analyzed by $16 \mathrm{~S}$-ribosomal RNA gene pyrosequencing. FEMS Microbiol Lett 351:187-194.

Sun L, Qiu F, Zhang X, Dai X, Dong X, Song W (2008) Endophytic bacterial diversity in rice (Oryza sativa L.) roots estimated by $16 \mathrm{~S}$ rDNA sequence analysis. Microb Ecol 55:415-424.

Toju H, Peay K G, Yamamichi M (2018) Core microbiomes for sustainable agroecosystems. Nature Plants.

Vega-Avila A D, Gumiere T, Andrade P A M (2015). Bacterial communities in the rhizosphere of Vitis vinifera L. cultivated under distinct agricultural practices in Argentina. Antonie van Leeuwenhoek.

Valenzuela-Aragon B, Parra-Cota F I, Santoyo G (2018). Plant-assisted selection: a promising alternative for in vivo identification of wheat (Triticum turgidum L. subsp. Durum) growth promoting bacteria. Plant and Soil.

Víctor J. Carrión, Perez-Jaramillo J, Cordovez V (2011). Pathogen-induced activation of diseasesuppressive functions in the endophytic root microbiome. Science.366. 
Wei V P, Friman T (2020). Rhizosphere immunity: targeting the underground for sustainable plant health management. Frontiers of Agricultural Science and Engineering. v.7(03):91-102.

Ye X, Seth D B, Ja M D (2015). Characterization of culturable bacterial endophytes and their capacity to promote plant growth from plants grown using organic or conventional practices. Frontiers in Plant Science.6.

Zhuang L B, Yu Y, Zhang N (2020). Synthetic community with six Pseudomonas strains screened from garlic rhizosphere microbiome promotes plant growth. Microbial Biotechnology.

Zhang J Y, Yong-Xin Liu\#, Xiaoxuan Guo, Yuan Qin, Ruben Garrido-Oter*, Paul Schulze-Lefert* \& Yang Bai*. (2021). High-throughput cultivation and identification of bacteria from the plant rootmicrobiota. Nature Protocols 1-16.

Zhang J.Y, Liu Y.X(2019). NRT1.1B is associated with root microbiota composition and nitrogen use in field-grown rice. Nature biotechnology.

Zhao S, Li L, Li S.H, Wang HF, Zhang YG, Wadaan MA, Li WJ, Tian CY (2015) Actinotalea suaeda sp.nov., isolated from the halophyte Suaeda physophora in Xinjiang,Northwest China. Anton Leeuw Int J G 107:1-7.

Zhao S, Zhou N, Zhao ZY (2016). High-Throughput Sequencing Analysis of the Endophytic Bacterial Diversity and Dynamics in Roots of the Halophyte Salicornia europaea. Current Microbiology.72(5):557.

\section{Figures}


Multy samples Rarefaction Curves

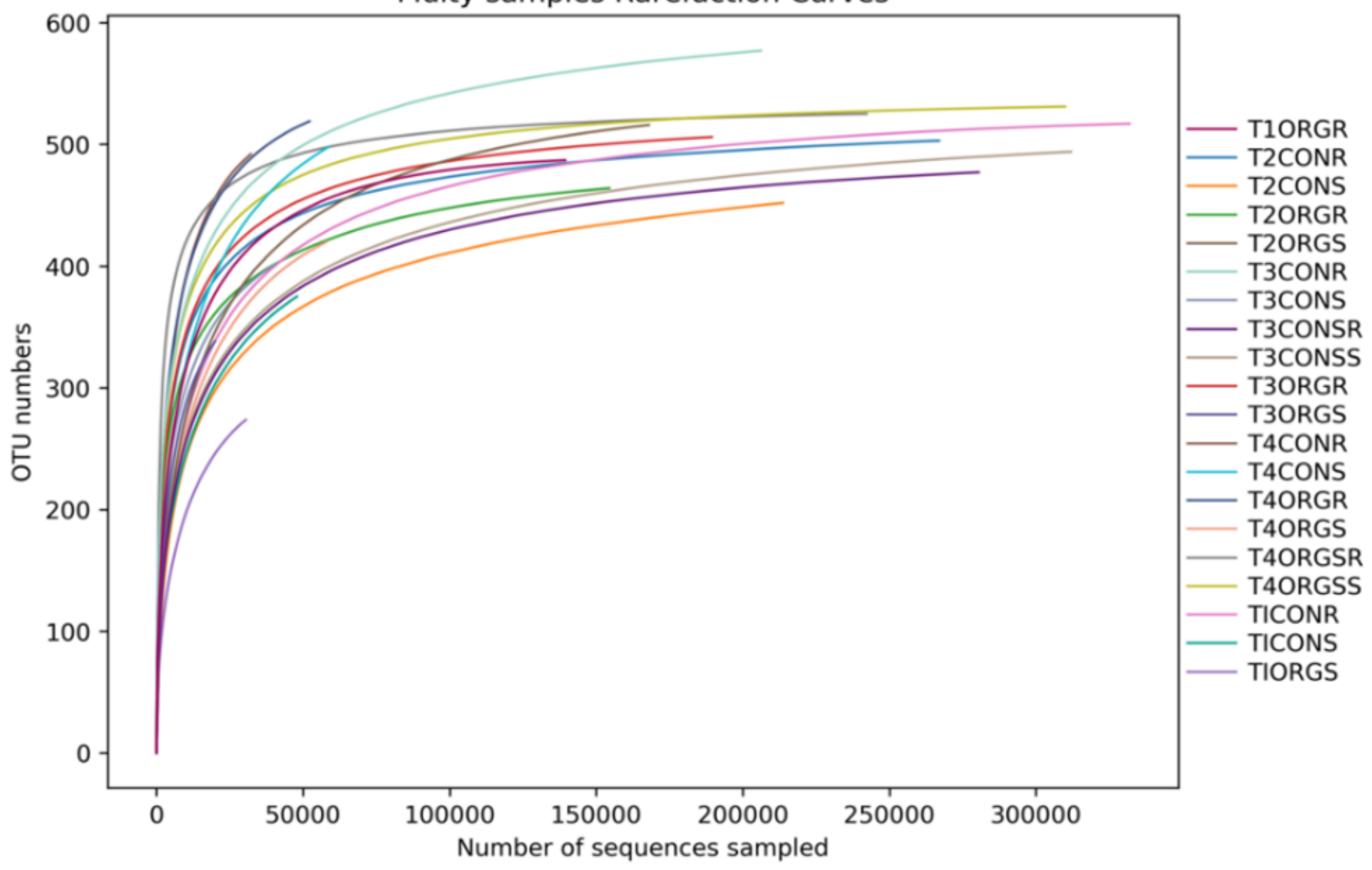

Figure 1

Rarefaction curves for bacterial OTUs, clustering at $97 \%$ rRNA sequence similarity 


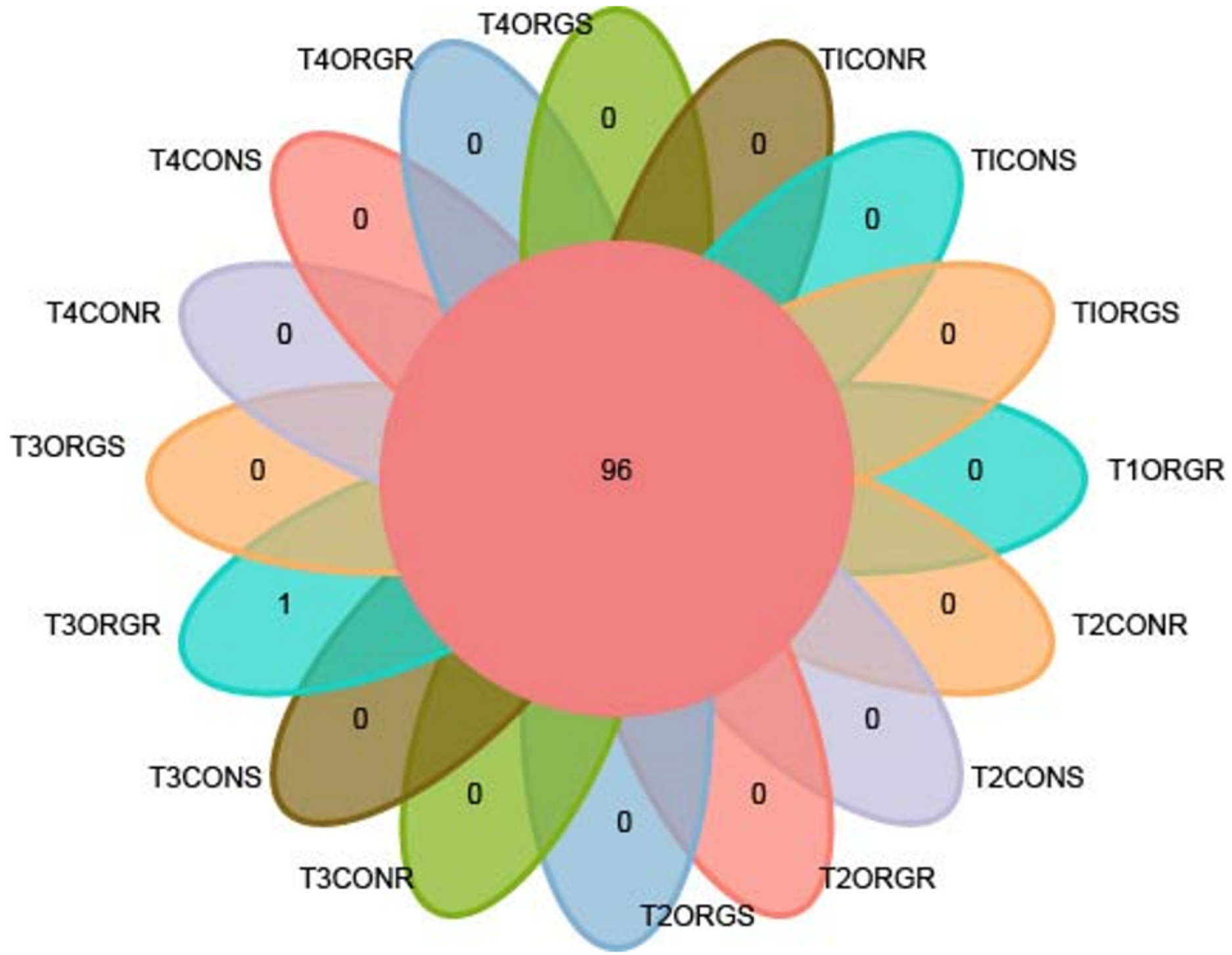

Figure 2

Venn diagram showing the shared OTUs among the 80 samples 

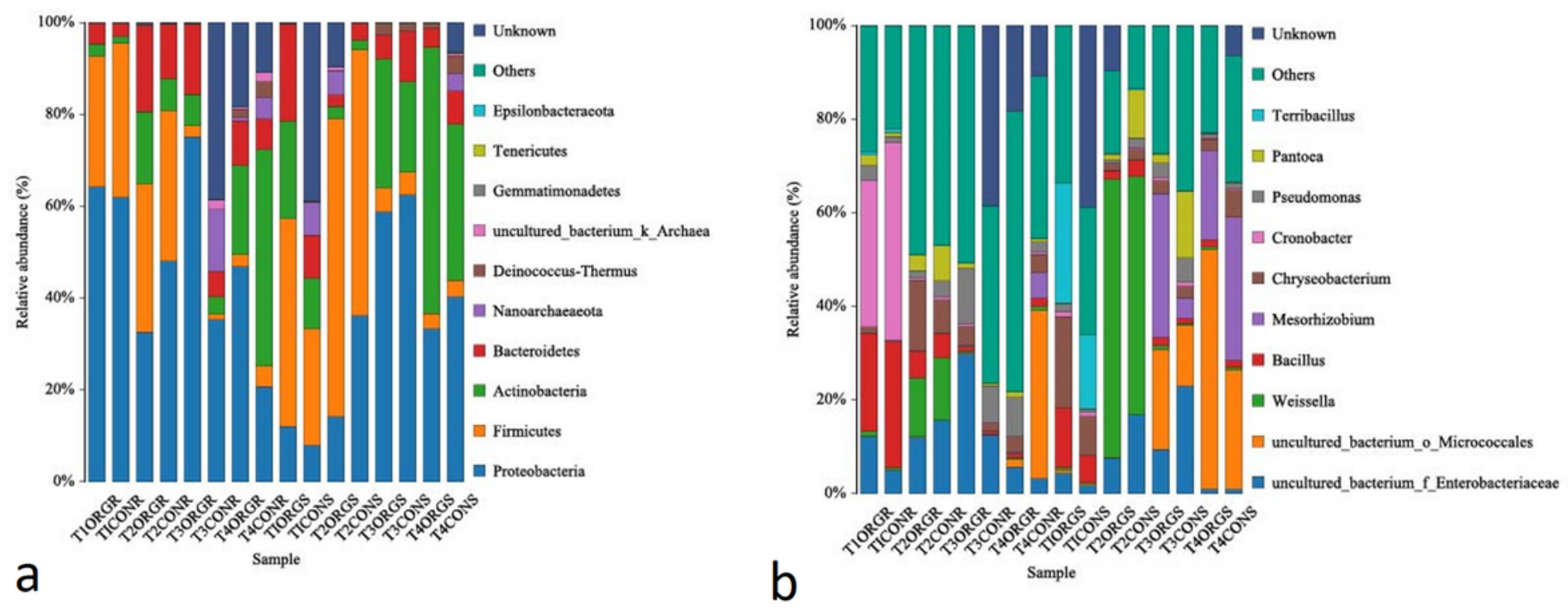

Figure 3

A Relative abundance of bacterial phylum of top 10 OTUs is shown in different groups. B Relative abundance of bacterial genera of top 10 OTUs is shown in different groups
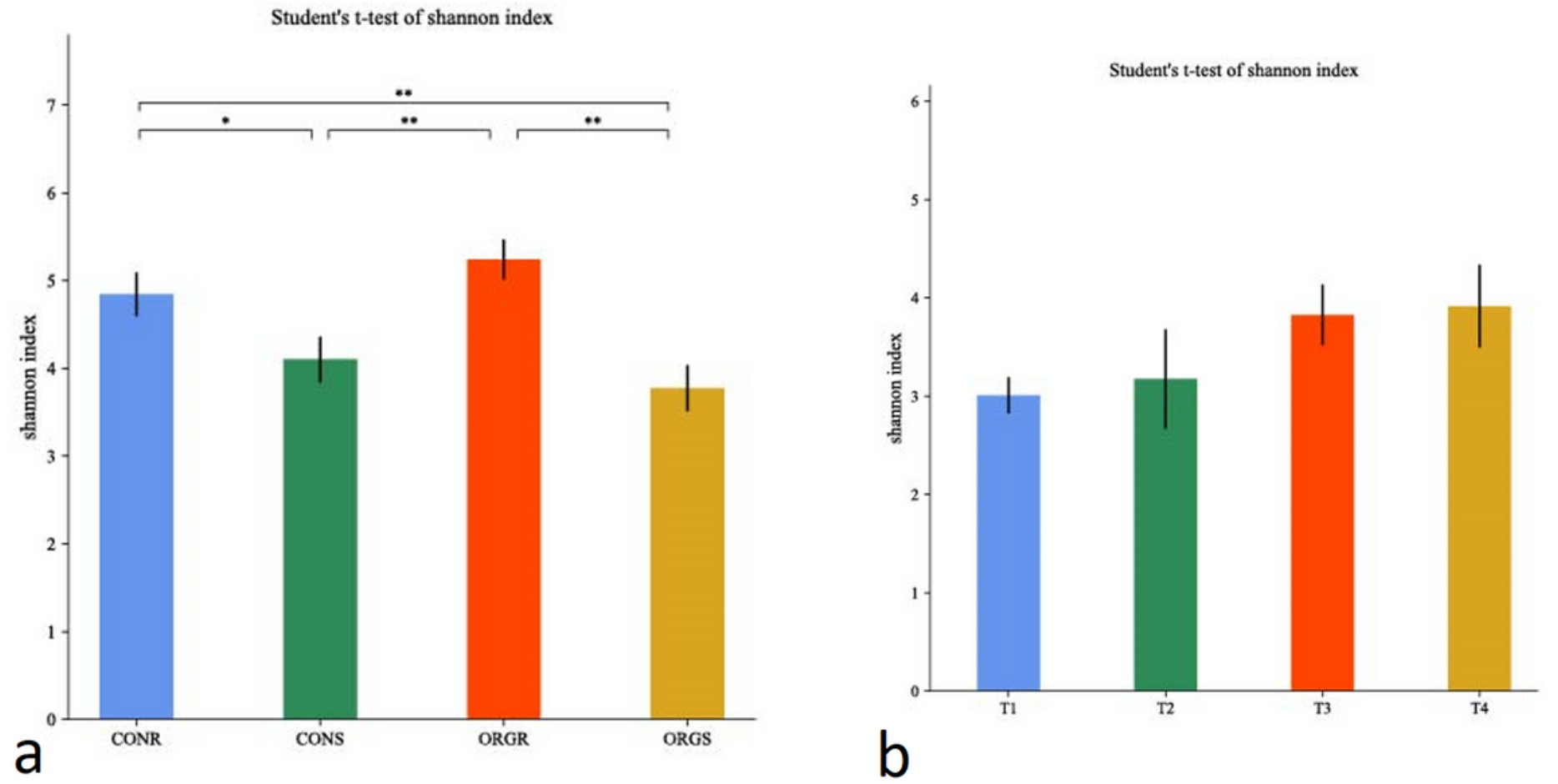

Figure 4

A. OTU bacterial Shannon diversity index for four different treatments. B. OTU bacterial Shannon diversity index for four different growth periods of tomato 

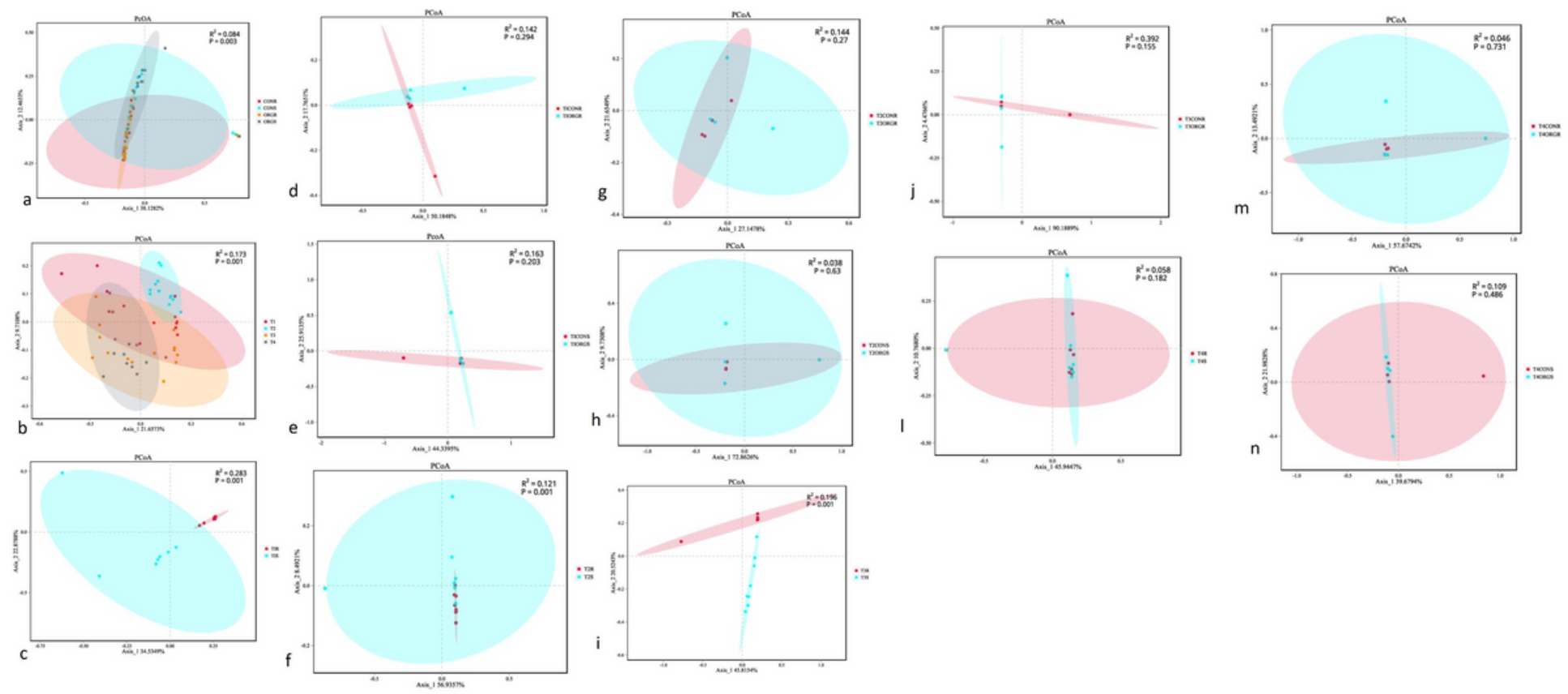

\section{Figure 5}

A. PCoA of bacterial microbiota using Bray-Curtis distance for different manipulation systems. B. PCoA of bacterial microbiota using Bray-Curtis distance for different periods. C. PCoA of bacterial microbiota using Bray-Curtis distance for different organ in seeding stage (TI). D. PCoA of root bacterial microbiota using Bray-Curtis distance for different manipulation in seeding stage (TI). E. PCoA of stem bacterial microbiota using Bray-Curtis distance for different manipulation in seeding stage (TI). F. PCoA of bacterial microbiota using Bray-Curtis distance for different organ in flowering stage (T2). G. PCoA of root bacterial microbiota using Bray-Curtis distance for different manipulation in flowering stage (T2). $\mathrm{H}$. PCoA of stem bacterial microbiota using Bray-Curtis distance for different manipulation in flowering stage (T2). I. PCoA of bacterial microbiota using Bray-Curtis distance for different organ in fruiting stage (T3). J. PCoA of root bacterial microbiota using Bray-Curtis distance for different manipulation in fruiting stage (T3). L. PCoA of root bacterial microbiota using Bray-Curtis distance for different manipulation in harvesting stage (T4). M. PCoA of root bacterial microbiota using Bray-Curtis distance for different manipulation in harvesting stage (T4). N. PCoA of stem bacterial microbiota using BrayCurtis distance for different manipulation in harvesting stage (T4).

\section{Supplementary Files}

This is a list of supplementary files associated with this preprint. Click to download.

- supplmentalTables.docx 